PROCEEDINGS OF THE

AMERICAN MATHEMATICAL SOCIETY

Volume 125, Number 6, June 1997, Pages 1755-1766

S 0002-9939(97)03708-8

\title{
MIXING PROPERTIES OF ONE-DIMENSIONAL CELLULAR AUTOMATA
}

\author{
RUNE KLEVELAND
}

(Communicated by Palle E. Jørgensen)

\begin{abstract}
We study a class of endomorphisms on the space of bi-infinite sequences over a finite set, and show that such a map is onto if and only if it is measure-preserving. A class of dynamical systems arising from these endomorphisms are strongly mixing, and some of them even $m$-mixing. Some of these are isomorphic to the one-sided shift on $\mathbb{Z}_{n}$ in both the topological and measure-theoretical sense. Such dynamical systems can be associated to $\mathcal{O}_{n}$, the Cuntz-algebra of order $n$, in a natural way.
\end{abstract}

\section{INTRODUCTION}

Shirvani and Rogers have proved in [Sh-Ro] that continuous maps $\prod_{-\infty}^{\infty} \mathbb{Z}_{2} \rightarrow$ $\prod_{-\infty}^{\infty} \mathbb{Z}_{2}$ that commute with the shift are surjective if and only if they are measurepreserving. We generalize this result to products over finite sets $\mathbb{L}=\mathbb{Z}_{S}$. We also generalize the mixing results from [Sh-Ro], and prove that some specific maps are $m$-mixing.

Theorem 2.1 states that we can obtain all continuous maps $\prod_{-\infty}^{\infty} \mathbb{L} \rightarrow \prod_{-\infty}^{\infty} \mathbb{L}$ that commute with the shift by extending maps $\mathbb{L}^{n} \rightarrow \mathbb{L}$. A nice result from [Hed], Theorem 2.2, states that the extended map is onto if and only if the extension to $\mathbb{L}^{n+m-1} \rightarrow \mathbb{L}^{m}$ is an $S^{n-1}-1$ map for all $m \in \mathbb{N}$. We replace the term "for all $m \in \mathbb{N}$ " with "for $m=\left(\begin{array}{c}2 S^{n-1}-1 \\ S^{n-1}\end{array}\right)$ ", thus we provide an algorithm for deciding ontoness of such maps.

This work is also inspired by [Mat], where some very specific endomorphisms are considered, and it is shown that the $C^{*}$-algebra generated by the continuous functions on $\prod_{-\infty}^{\infty}\{0,1\}$ and the isometry induced by the endomorphism is the Cuntz-algebra $\mathcal{O}_{4}$. We extend this result.

\section{TWO BASIC STRUCTURE THEOREMS}

Let $S \in\{2,3, \ldots\}$, and let $\mathbb{L}=\{0,1, \ldots, S-1\}$ be the set of $S$ symbols with discrete topology and normalized Haar-measure $\dot{\mu}$, that is, $\dot{\mu}(\{a\})=S^{-1}$. A biinfinite sequence over $\mathbb{L}$ is a function $\mathbb{Z} \rightarrow \mathbb{L}$.

Let $\mathbb{L}^{\infty}=\prod_{-\infty}^{\infty} \mathbb{L}$ be the family of bi-infinite sequences over $\mathbb{L}$ equipped with the product topology $\tau$ and the product measure $\mu$ defined by $\dot{\mu}$. It is well known that $\mathbb{L}^{\infty}$ is a compact, totally disconnected perfect metric space, and hence homeomorphic to the Cantor discontinum.

Received by the editors October 23, 1995 and, in revised form, December 13, 1995.

1991 Mathematics Subject Classification. Primary 47A35, 22D25; Secondary 28D05, 46L05. 
Let $C\left(\mathbb{L}^{n}, \mathbb{L}\right)$ be the set of maps $\mathbb{L}^{n} \rightarrow \mathbb{L}$. If $f \in C\left(\mathbb{L}^{n}, \mathbb{L}\right)$, define a map $f_{m}: \mathbb{L}^{m+n-1} \rightarrow \mathbb{L}^{m}$ by $f_{m}(x)_{i}=f\left(x_{i}, x_{i+1}, \ldots, x_{i+n-1}\right)$. In addition, define a map $f_{\infty}: \mathbb{L}^{\infty} \rightarrow \mathbb{L}^{\infty}$ by $f_{\infty}(x)_{i}=f\left(x_{i}, x_{i+1}, \ldots, x_{i+n-1}\right)$. Let $\sigma$ be the left-shift on $\mathbb{L}^{\infty}$, that is, $\sigma(x)_{i}=x_{i+1}$.

Theorem 2.1 ([Hed, Theorem 3.1 and 3.4]). If $f \in C\left(\mathbb{L}^{n}, \mathbb{L}\right)$, then $f_{\infty}$ is continuous and $f_{\infty}$ commutes with the shift $\sigma$. Conversely, for every continuous map $\psi: \mathbb{L}^{\infty} \rightarrow \mathbb{L}^{\infty}$ that commutes with $\sigma$, there exists $n \in \mathbb{N}, f \in C\left(\mathbb{L}^{n}, \mathbb{L}\right)$ and $k \in \mathbb{N}$ such that $\psi=f_{\infty} \circ \sigma^{k}$.

This theorem shows that the set of continuous maps $\mathbb{L}^{\infty} \rightarrow \mathbb{L}^{\infty}$ which commute with the shift is countable, since $\operatorname{Card} C\left(\mathbb{L}^{n}, \mathbb{L}\right)=S^{S^{n}}$. We will mainly consider the surjective maps that commute with the shift.

Theorem 2.2 ([Hed, Theorem 5.1 and 5.4]). Let $f \in C\left(\mathbb{L}^{n}, \mathbb{L}\right)$. Then the following conditions are equivalent:

i) $f_{\infty}$ is surjective.

ii) $f_{m}$ is surjective for all $m \in \mathbb{N}$.

iii) $f_{m}$ is a $S^{n-1}-1$ map for all $m \in \mathbb{N}$.

If $g_{\infty}=f_{\infty} \circ \sigma^{r}$ for some $f \in C\left(\mathbb{L}^{s-r}, \mathbb{L}\right)$, we will use the sloppy notation $g_{\infty}=f\left(x_{r}, \ldots, x_{s}\right)$.

An $n$-block over $\mathbb{L}$ is an element in $\mathbb{L}^{n}$. The length $n$ of an $n$-block $x$ is denoted by $|x|$. Let $x=x_{1} x_{2} \ldots x_{n}$ be an $n$-block over $\mathbb{L}$. Define

$$
\operatorname{Cyl}(x, p)=\left\{y \in \mathbb{L}^{\infty}: y_{p} y_{p+1} \ldots y_{p+n-1}=x\right\} .
$$

The length of a cylinder $\operatorname{Cyl}(x, p)$ is the length of the block $x$. It is evident that $\operatorname{Cyl}(x, p)$ is a clopen set with $\mu(\operatorname{Cyl}(x, p))=S^{-|x|}$.

Let $A \subseteq \mathbb{L}^{\infty}$ be a measurable set. We say that the support of $A$, denoted $\operatorname{Supp}(A)$, is contained in a subset $\tilde{A}$ of $\mathbb{Z}$ and write $\operatorname{Supp}(A) \subseteq \tilde{A}$ if $A$ is a member of the least $\sigma$-algebra that contains every cylinder set $\operatorname{Cyl}(a, p)$ where $p \in \tilde{A}$ and $a \in \mathbb{L}$. Note that we do not require $\operatorname{Supp}(A)$ to be an explicit subset of $\mathbb{Z}$, even if this might be possible. If two sets $A, B \in \Omega$ have disjoint support, then $\mu(A \cap B)=\mu(A) \mu(B)$, since $\mu$ is a product measure.

Let $f_{\infty}$ defined by $f \in C\left(\mathbb{L}^{n}, \mathbb{L}\right)$ be surjective, and let $x \in \mathbb{L}^{m}$ be an $m$-block. By Theorem 2.2 we conclude that $f_{\infty}^{-1}(\mathrm{Cyl}(x, p))$ is a union of $S^{n-1}$ cylinders $\operatorname{Cyl}\left(y^{(\kappa)}, p\right)$ where $y^{(\kappa)} \in \mathbb{L}^{n+m-1}$ and $\kappa \in \mathbb{L}^{n-1}$, since $f_{|x|}$ is a $S^{n-1}-1$ map. Thus we have

$$
f_{\infty}^{-1}(\operatorname{Cyl}(x, p))=\bigcup_{\kappa \in \mathbb{L}^{n-1}} \operatorname{Cyl}\left(y^{(\kappa)}, p\right) .
$$

Let $g_{\infty}=f_{\infty} \circ \sigma^{r}=f\left(x_{r}, \ldots, x_{s}\right)$. Since $f_{\infty}$ commutes with $\sigma$ we have

$$
g_{\infty}^{-1}(\operatorname{Cyl}(x, p))=f_{\infty}^{-1} \circ \sigma^{-r}(\operatorname{Cyl}(x, p))=\bigcup_{\kappa \in \mathbb{L}^{s-r}} \operatorname{Cyl}\left(y^{(\kappa)}, p+r\right) .
$$

From this observation we obtain the important inclusion

$$
\operatorname{Supp}\left(g_{\infty}^{-1}(\operatorname{Cyl}(x, p))\right) \subseteq[p+r, p+s+|x|-1] .
$$




\section{The MAPS $f_{\infty}$ DEFINED By PERMUtATIVE $f$}

It is possible to describe a class of surjective $f_{\infty}$ in terms of properties of $f$.

Definition 3.1. Let $f_{\infty}=f\left(x_{r}, \ldots, x_{s}\right)$. We say that $f$ is permutative in $x_{j}$ if the map $g_{x_{r} \ldots x_{j-1} x_{j+1} \ldots x_{s}}: \mathbb{L} \rightarrow \mathbb{L}$ given by

$$
g_{x_{r} \ldots x_{j-1} x_{j+1} \ldots x_{s}}\left(x_{j}\right)=f\left(x_{r}, \ldots, x_{s}\right)
$$

is a permutation for all $x_{r} \ldots x_{j-1} x_{j+1} \ldots x_{s} \in \mathbb{L}^{s-r-1}$.

Theorem 3.2 ([Hed, Theorem 6.6 and Theorem 6.7]). Let $f_{\infty}=f\left(x_{r}, \ldots, x_{s}\right)$. If $f$ is permutative in the first or the last variable, then $f_{\infty}$ is surjective. If $f$ is permutative in both the first and the last variable, then $f_{\infty}$ is a $S^{s-r}-1$ map.

Proof. Suppose $f\left(x_{r}, \ldots, x_{s}\right)$ is permutative in $x_{s}$. We show that $f_{m}$ is a $S^{s-r}-1$ map. Let $\kappa \in \mathbb{L}^{s-r}$ and $a \in \mathbb{L}$. Since $f$ is permutative in $x_{s}$, the map $g_{\kappa}: \mathbb{L} \rightarrow \mathbb{L}$ given by $g_{\kappa}(a)=f(\kappa a)$ is a permutation of $\mathbb{L}$, which implies that, for each $\kappa \in \mathbb{L}^{s-r}$, there is a unique $a_{\kappa} \in \mathbb{L}$ such that $f\left(\kappa a_{\kappa}\right)=b$. Since Card $\mathbb{L}^{s-r}=S^{s-r}, f=f_{1}$ is a $S^{s-r}-1$ map.

Assume that Card $f_{m}^{-1}(x)=k$. Choose $y^{(j)} \in \mathbb{L}^{m+s-r}$ such that $f_{m}^{-1}(x)=$ $\left\{y^{(1)}, y^{(2)}, \ldots, y^{(k)}\right\}$. Let $a \in \mathbb{L}$. If $f$ is permutative in $x_{s}$, there exists, for each $j$, a unique $a_{j} \in \mathbb{L}$ such that $f_{m+1}\left(y^{(j)} a_{j}\right)=x a$. It follows that $f_{m+1}^{-1}(x a)=$ $\left\{y^{(1)} a_{1}, y^{(2)} a_{2}, \ldots, y^{(k)} a_{k}\right\}$, thus Card $f_{m+1}^{-1}(x a)=k$. By induction on $m, f_{m}$ is a $S^{s-r}-1$ map for all $m \in \mathbb{N}$. By Theorem $2.2, f_{\infty}$ is surjective. The proof is analogous if $f$ is permutative in $x_{r}$.

Suppose $f$ is permutative in both $x_{r}$ and $x_{s}$, and that $f_{\infty}(x)=y$. If $\kappa=$ $x_{1} x_{2} \ldots x_{s-r} \in \mathbb{L}^{s-r}$ is given, the argument above shows that $x_{s-r+1}, x_{s-r+2}, \ldots$ are uniquely determined by $y$, and by symmetry $x_{r-1}, x_{r-2}, \ldots$ also are uniquely determined. This implies that Card $f_{\infty}^{-1}(y) \leq S^{s-r}$. But each $\kappa=x_{1} x_{2} \ldots x_{s-r} \in$ $\mathbb{L}^{s-r}$ gives rise to $x \in f_{\infty}^{-1}(y)$, thus Card $f_{\infty}^{-1}(y)=S^{s-r}$.

Remark 3.3. The conclusion of the first part of Theorem 3.2 cannot be obtained if $f$ is permutative in some other variable than the first or last one. A simple counterexample for $S=2$ is provided by $f(a, b, c, d)=a b d+c \bmod 2$. It is easy to check that Card $f_{2}^{-1}(0,0)=9 \neq 2^{3}$, thus $f_{\infty}$ is not surjective by Theorem 2.2.

Remark 3.4. It is possible to show that if $f_{\infty}=f\left(x_{r}, \ldots, x_{s}\right)$ is a $S^{s-r}-1$ map, then $f$ is permutative in both $x_{r}$ and $x_{s}$. This is Theorem 17.2 in [Hed].

Remark 3.5. Let $S=2$. Theorem 3.2 implies that $g_{\infty}$ and $h_{\infty}$ defined by

$$
g(a, b, c)=a b+c \bmod 2 \quad \text { and } \quad h(a, b, c)=a+b c \bmod 2
$$

are surjective. This implies that $f_{\infty}=g_{\infty} \circ h_{\infty}$ defined by

$$
\begin{aligned}
f(a, b, c, d) & =g(h(a, b, c), h(b, c, d), h(c, d, e)) \\
& =(a+b c)(b+c d)+c+d e \\
& =a b+a c d+b c+b c d+d e \bmod 2
\end{aligned}
$$

is surjective. But this $f$ is not covered by Theorem 3.2, so the result is not complete.

Remark 3.6. The construction in the proof of Theorem 3.2 tells us something about the structure of the inverse image of a cylinder under $f_{\infty}$. Let $f \in C\left(\mathbb{L}^{s-r}, \mathbb{L}\right)$ be permutative in the last variable, and let $x \in \mathbb{L}^{m}$. We saw that for each $\kappa \in \mathbb{L}^{s-r}$ 
there exist $y^{(\kappa)} \in f_{m}^{-1}(x)$ such that $\kappa=y_{1}^{(\kappa)} y_{2}^{(\kappa)} \ldots y_{s-r}^{(\kappa)}$. If we index the set $f_{m}^{-1}(x)$ over $\mathbb{L}^{s-r}$, we can write $f_{m}^{-1}(x)=\bigcup_{\kappa \in \mathbb{L}^{s-r}} y^{(\kappa)}$.

Let $p \in \mathbb{Z}$. This implies that if $f_{\infty}=f\left(x_{r}, \ldots, x_{s}\right)$ and $f$ is permutative in $x_{s}$, the $S^{s-r}$ cylinders in $f_{\infty}^{-1}(\operatorname{Cyl}(x, p))$ can be indexed over $\mathbb{L}^{s-r}$ such that

$$
f_{\infty}^{-1}(\operatorname{Cyl}(x, p))=\bigcup_{\kappa \in \mathbb{L}^{s-r}} \operatorname{Cyl}\left(y^{(\kappa)}, p+r\right), \quad \kappa=y_{1}^{(\kappa)} y_{2}^{(\kappa)} \ldots y_{s-r}^{(\kappa)}
$$

If $f$ is permutative in both $x_{r}$ and $x_{s}$, each $y^{(\kappa)}$ is uniquely determined by $y_{k+1}^{(\kappa)} y_{k+2}^{(\kappa)} \ldots y_{k+s-r}^{(\kappa)}$ if $0 \leq k \leq m$. This implies that, if

$$
\operatorname{Supp}(\operatorname{Cyl}(\kappa, q)) \subseteq\left[p+r, p+s+\left|y^{(\kappa)}\right|-1\right] \supseteq \operatorname{Supp}\left(\operatorname{Cyl}\left(y^{(\kappa)}, p+r\right)\right),
$$

which is equivalent to $p+r \leq q \leq p+r+|x|$, then

$$
f_{\infty}^{-1}(\operatorname{Cyl}(x, p)) \cap \operatorname{Cyl}(\kappa, q)=\operatorname{Cyl}(z, p+r)
$$

for some $z \in \mathbb{L}^{|x|+s-r}$. This observation is used in the proof of Theorem 7.1.

\section{Which $f \in C\left(\mathbb{L}^{n}, \mathbb{L}\right)$ Defines A SURJECtIVE $f_{\infty}$ ?}

Using Theorem 2.2, one can show that many of the maps $f_{\infty}$ are not surjective. But this theorem does not give a general algorithm for deciding if a particular $f$ give rise to a surjective $f_{\infty}$, since it offers no restriction on $m$.

For a non-surjective $f_{\infty} \in C\left(\mathbb{L}^{n}, \mathbb{L}\right)$, let $m(f)=\min \left\{m \in \mathbb{N}: f_{m}\right.$ is not $\left.S^{n-1}-1\right\}$, and let

$$
\rho_{S}(n)=\max \left\{m(f): f \in C\left(\mathbb{L}^{n}, \mathbb{L}\right), f_{\infty} \text { is not surjective }\right\} .
$$

Since Card $C\left(\mathbb{L}^{n}, \mathbb{L}\right)$ is finite for all $n \in \mathbb{N}, \rho_{S}(n) \leq \infty$ for all $n \in \mathbb{N}$. If $f \in C\left(\mathbb{L}^{n}, \mathbb{L}\right)$ and $f_{\rho_{S}(n)}$ is $S^{n-1}-1$, then $f_{\infty}$ is surjective. This implies that condition iii) in Theorem 2.2 can be replaced by "for all $m \leq \rho_{S}(n)$ ". But if $f_{m}$ is a $S^{n-1}-1$ map, then $f_{m-1}$ is also has this property by a simple counting argument. Thus the condition can be relaxed to "for $m=\rho_{S}(n)$ ".

In [Sh-Ro] it is claimed (at least in remark 2.5) that $\rho_{2}(n) \leq n$. This is true for $n \leq 3$, since we for $n \leq 3$ have that every $f \in C\left(\mathbb{L}^{n}, \mathbb{L}\right)$ such that $f_{n}$ is a $2^{n-1}-1$ map turns out to be permutative in either the first or last variable, and hence surjective by Theorem 3.2. But $\rho_{2}(n)>n$ for $n=4$ and $n=5$. We have a particular $f \in C\left(\mathbb{L}^{4}, \mathbb{L}\right)$ defined by

$$
f(a, b, c, d)=(1+a)(1+d)+d(b+a c) \bmod 2
$$

with the property that $f_{4}: \mathbb{L}^{7} \rightarrow \mathbb{L}^{4}$ is a $2^{4-1}-1$ map. But $f_{5}: \mathbb{L}^{8} \rightarrow \mathbb{L}^{5}$ is not a $2^{4-1}-1$ map, thus $\rho_{2}(4) \geq 5$. In addition we have an $f \in C\left(\mathbb{L}^{5}, \mathbb{L}\right)$ defined by

$$
f(a, b, c, d, e)=a(b(d+e)+c)+e \bmod 2 .
$$

This function has the property that $f_{6}: \mathbb{L}^{10} \rightarrow \mathbb{L}^{6}$ is a $2^{5-1}-1$ map, but $f_{7}: \mathbb{L}^{11} \rightarrow$ $\mathbb{L}^{7}$ is not a $2^{5-1}-1$ map. Thus $\rho_{2}(5) \geq 7$. The function in equation (4.3) is quite rare, and was found by a computer.

To obtain a good estimate for $\rho_{S}(n)$ is to my knowledge an open problem. I will now provide a bad one.

A configuration of order $n$ is a function $\psi: \mathbb{L}^{n-1} \rightarrow \mathbb{N} \cup\{0\}$. Let $\|\psi\|$ be the one-norm, that is, $\|\psi\|=\sum_{\kappa \in \mathbb{L}^{n-1}} \psi(\kappa)$. Let $\mathbf{1}$ be the configuration defined by $\mathbf{1}(\kappa)=1$. 
Let $f \in C\left(\mathbb{L}^{n}, \mathbb{L}\right)$ be fixed. For $x \in \mathbb{L}^{m}$, define the configuration $\mathbf{1}_{x}$ by

$$
\mathbf{1}_{x}(\kappa)=\operatorname{Card}\left\{y \in f_{m}^{-1}(x): y_{m+1} y_{m+2} \ldots y_{m+n-1}=\kappa\right\} .
$$

It is obvious that $\left\|\mathbf{1}_{x}\right\|=\operatorname{Card} f_{|x|}^{-1}(x)$. We call $\psi$ legal if $\|\psi\|=S^{n-1}$ and possible if there exist $m \in \mathbb{N}$ and $x \in \mathbb{L}^{m}$ such that if $\psi=\mathbf{1}_{x}$. Theorem 2.2 implies that $f_{\infty}$ is surjective if and only if every possible configuration is legal.

Lemma 4.1. The number of legal configurations corresponding to a function $f \in$ $C\left(\mathbb{L}^{n}, \mathbb{L}\right)$ is less than or equal to $\left(\begin{array}{c}2 S^{n-1}-1 \\ S^{n-1}\end{array}\right)$.

Proof. There is a bijective correspondence between the legal configurations of order $n$ and finite sequences of length $2 S^{n-1}-1$ consisting of $S^{n-1}$ ones and Card $\mathbb{L}^{n-1}-$ $1=S^{n-1}-1$ zeros. To a configuration $\psi$ we associate the finite sequence consisting of $\psi(0)$ ones, one zero, $\psi(1)$ ones, one zero and so on. Then each legal configuration is associated to a unique sequence with the given property, and it is fairly easy to see that each such sequence gives a legal configuration. Thus the number of legal configurations is the number of legal sequences, that is, $\left(\begin{array}{c}2 S^{n-1}-1 \\ S^{n-1}\end{array}\right)$.

We define the depth of a possible configuration $\psi$ as the least $m \in \mathbb{N}$ such that $\psi=\mathbf{1}_{x}$ for some $x \in \mathbb{L}^{m}$, with the convention that the depth of $\mathbf{1}$ is 0 .

Theorem 4.2. Let $a(m)$ be the number of possible configurations with depth less than or equal to $m$.

i) If $a(m+1)=a(m)$, then $a(m+k)=a(m)$ for all $k \in \mathbb{N}$.

ii) If $a(m+1)>a(m)$, then $a(m)>m$.

iii) If $f_{m}$ is an $S^{n-1}-1$ map and $a(m+1)=a(m)$, then $f_{\infty}$ is surjective.

iv) If $f_{m}$ is an $S^{n-1}-1$ map for $m=\left({ }^{2 S^{n-1}-1} S^{n-1}\right)$, then $f_{\infty}$ is surjective, thus $\rho_{S}(n) \leq$ $\left(\begin{array}{c}2 S^{n-1}-1 \\ S^{n-1}\end{array}\right)$.

Proof.

i) Suppose that $a(m+2)>a(m+1)$. Then there is a configuration $\varphi_{x b}$ where $x \in \mathbb{L}^{n+1}$ and $b \in \mathbb{L}$ with depth $m+2$. But this implies that the depth of $\varphi_{x}$ is $m+1$, a contradiction. The statement follows by induction on $m$.

ii) If $a(m+1)>a(m)$, then $a(k+1)>a(k)$ for all $k \leq m$ by the first statement, thus $a(m)>m$, since $a(0)=1$.

iii) Since $a(m+1)=a(m)$, every possible configuration has depth less or equal to $m$ by i), thus every possible configuration is legal, since the assumption that $f_{m}$ is a $S^{n-1}-1$ map implies that $f_{m^{\prime}}$ is a $S^{n-1}-1$ map for $m^{\prime} \leq m$.

iv) Let $m=\left(\begin{array}{c}2 S^{n-1}-1 \\ S^{n-1}\end{array}\right)$. If $f_{\infty}$ is not surjective, then $a(m+1)>a(m)$ thus there would exist at least $a(m)>m$ legal configurations. This contradicts Lemma 4.1.

Part iv) in the above theorem gives an upper bound for the function $\rho_{S}(n)$, but this is certainly not an optimal result. One way to improve it is to consider the growing rate of $a(m)$. Another could be a complete change of strategy, and the solution might be simple ....

Theorem 4.2 above gives an algorithm for deciding if a particular $f \in C\left(\mathbb{L}^{n}, \mathbb{L}\right)$ gives rise to a surjective $f_{\infty}$. We calculate the number $a(m)$ until $a(m+1)=a(m)$ or until we find an illegal configuration. One of these events has to occur before $\max (a(m), m) \geq\left(\begin{array}{c}2 S^{n-1}-1 \\ S^{n-1}\end{array}\right)$. In the first case $f_{\infty}$ is surjective; in the second it is not. 


\section{Measure-preserving properties of $f_{\infty}$}

The part i) $\Leftrightarrow$ iii) of Theorem 2.2 in fact states that $f_{\infty}$ is surjective if and only if $f_{m}$ is measure-preserving for all $m \in \mathbb{N}$. The next theorem extends this result.

Theorem 5.1. Suppose $\psi$ is a continuous map $\mathbb{L}^{\infty} \rightarrow \mathbb{L}^{\infty}$ that commutes with the shift $\sigma$. Then $\psi$ is onto if and only if $\psi$ is measure-preserving.

Proof. Let $\psi$ be measure-preserving, and put $A=\psi\left(\mathbb{L}^{\infty}\right)$. Since $\mathbb{L}^{\infty}$ is compact, $A$ is compact and hence closed. In addition

$$
\mu(A)=\mu\left(\psi^{-1}(A)\right)=\mu\left(\mathbb{L}^{\infty}\right)=1,
$$

thus $A^{\complement}$ is open with zero measure, hence empty.

Assume $\psi$ is onto. Theorem 2.1 implies that $\psi=f_{\infty}=f\left(x_{r}, \ldots, x_{s}\right)$. Theorem 1.1 in [Wal] implies that $f_{\infty}$ is measure-preserving if it is measure-preserving for every set in the elementary family that generates the Borel $\sigma$-algebra on $\mathbb{L}^{\infty}$, that is, all the cylinder-sets $A_{p}=\operatorname{Cyl}\left(x^{(p)},-p\right)$ where $p \in \mathbb{N}$ and $x^{(p)} \in \mathbb{L}^{2 p+1}$. Now $\mu\left(A_{p}\right)=S^{-(2 p+1)}$. Equation $(2.3)$ says that $f_{\infty}^{-1}\left(A_{p}\right)$ is a union of exactly $S^{s-r}$ cylinders, each of length $2 p+1+s-r$ and hence measure $S^{-(2 p+1+s-r)}$. It follows that

$$
\mu\left(f_{\infty}^{-1}\left(A_{p}\right)\right)=S^{s-r} \cdot S^{-(2 p+1+s-r)}=S^{-(2 p+1)}=\mu\left(A_{p}\right),
$$

thus $\psi=f_{\infty}$ is measure-preserving.

Remark 5.2. The above theorem can also be found in [Sh-Ro, Theorem 2.4], but only for $S=2$, and with a different line of proof. The authors of this article claim to have proven two more equivalent conditions, but their use of this result gives a false conclusion, as shown at the start of section 4 .

In Theorem 5.1 we assume that $\psi$ commutes with the shift. One might ask if something similar can be obtained for continuous $\psi$ that does not have this property. Observing that $\mathbb{L}^{\infty}$ in fact is a compact group, we can apply the following nice theorem, taken from an example in [Wal]:

Theorem 5.3. Let $\mathcal{G}$ be a compact Abelian group equipped with the Haar-measure $\mu$. Suppose $\psi$ is a continuous, surjective and additive map $\mathcal{G} \rightarrow \mathcal{G}$. Then $\psi$ is measure-preserving.

Proof. Define a measure $\nu$ on $\mathcal{G}$ by $\nu(E)=\mu\left(\psi^{-1}(E)\right)$ for all measurable $E \subseteq \mathcal{G}$. Then $\nu(\mathcal{G})=1$ and we have

$$
\nu(\psi(x)+E)=\mu\left(\psi^{-1}(\psi(x)+E)\right) \stackrel{(*)}{=} \mu\left(x+\psi^{-1}(E)\right)=\mu\left(\psi^{-1}(E)\right)=\nu(E) .
$$

(*) follows from the additivity of $\psi$. This shows that $\nu$ is a rotation-invariant probability measure, since $\psi$ is surjective, thus it is the unique Haar-measure $\mu$. It follows that $\psi$ is measure-preserving.

Remark 5.4. Let $\left(a_{j}\right)_{j \in \mathbb{Z}}$ be a bi-infinite sequence over $\mathbb{L}$ with the property that $\operatorname{gcd}\left(S, a_{j}, a_{j+1}\right)=1$ and $a_{j}$ or $a_{j+1}$ or both are nonzero. Define a transformation $\psi: \mathbb{L}^{\infty} \rightarrow \mathbb{L}^{\infty}$ by

$$
\psi(x)_{i}=\left\{\begin{array}{lll}
\sum_{j=0}^{2 i+1} a_{j} x_{j} & \bmod S & \text { for } i \geq 0 \\
\sum_{j=2 i}^{-1} a_{j} x_{j} & \bmod S & \text { for } i<0
\end{array}\right.
$$


It is not difficult to verify that this map satisfies the requirements of Theorem 5.3. Since there are uncountably many sequences $\left(a_{j}\right)_{j \in \mathbb{Z}}$ that satisfy the conditions for every $S \geq 2$, there are uncountably many measure-preserving maps $\mathbb{L}^{\infty} \rightarrow \mathbb{L}^{\infty}$. But only a countable subset of these commutes with the shift $\sigma$ by Theorem 2.1.

\section{MiXing}

The following lemma generalizes Theorem 5.1 for $f_{\infty}=f\left(x_{r}, \ldots, x_{s}\right)$ where $f$ is permutative in $x_{r}$ of $x_{s}$. It will be used to prove mixing-properties.

Lemma 6.1. Let $x \in \mathbb{L}^{m}, k \in \mathbb{Z}, A=\operatorname{Cyl}(x, p)$ and $f_{\infty}=f\left(x_{r}, \ldots, x_{s}\right)$ where $f$ is permutative in $x_{s}$, respectively $x_{r}$. If $B \subseteq \mathbb{L}^{\infty}$ is a measurable set and $\operatorname{Supp}(B) \subseteq$ $(-\infty, p+s-1] \cup[p+s+m, \infty)$, respectively $\operatorname{Supp}(B) \subseteq(-\infty, p+r-1] \cup[p+r+m, \infty)$, then $\mu\left(f_{\infty}^{-1}(A) \cap B\right)=\mu(A) \mu(B)$.

Proof. Assume that $f$ is permutative in $x_{s}$. By equation (3.4) we have

$$
f_{\infty}^{-1}(A)=f_{\infty}^{-1}(\operatorname{Cyl}(x, p))=\bigcup_{\kappa \in \mathbb{L}^{n-1}} \operatorname{Cyl}\left(y^{(\kappa)}, p+r\right), \quad \kappa=y_{1}^{(\kappa)} y_{2}^{(\kappa)} \ldots y_{m-1}^{(\kappa)},
$$

where $y^{(\kappa)} \in \mathbb{L}^{m+s-r}$. This implies that $f_{\infty}^{-1}(A) \cap \operatorname{Cyl}(\kappa, p+r)=\operatorname{Cyl}\left(y^{(\kappa)}, p+r\right)$.

For every $\kappa \in \mathbb{L}^{s-r}$, define $B_{\kappa}=B \cap \operatorname{Cyl}(\kappa, p+r)$. Then $B=\bigcup_{\kappa \in \mathbb{L}^{s-r}} B_{\kappa}$ and

$$
\begin{aligned}
\mu\left(f_{\infty}^{-1}(A) \cap B\right) & =\mu\left(\left(\bigcup_{\kappa \in \mathbb{L}^{s-r}} \operatorname{Cyl}\left(y^{(\kappa)}, p+r\right)\right) \cap\left(\bigcup_{\kappa \in \mathbb{L}^{s-r}} B_{\kappa}\right)\right) \\
& =\mu\left(\bigcup_{\kappa \in \mathbb{L}^{s-r}} B_{\kappa} \cap \operatorname{Cyl}\left(y^{(\kappa)}, p+r\right)\right) \\
& =\sum_{\kappa \in \mathbb{L}^{s-r}} \mu\left(B_{\kappa} \cap \operatorname{Cyl}\left(y^{(\kappa)}, p+r\right)\right) \\
& =\sum_{\kappa \in \mathbb{L}^{s-r}} \mu\left(B_{\kappa} \cap \operatorname{Cyl}\left(y_{1+s-r}^{(\kappa)} y_{2+s-r}^{(\kappa)} \ldots y_{m+s-r}^{(\kappa)}, p+s\right)\right) \\
& \stackrel{(*)}{=} \sum_{\kappa \in \mathbb{L}^{s-r}} \mu\left(B_{\kappa}\right) \cdot S^{-m}=\mu(A) \mu(B) .
\end{aligned}
$$

$(*)$ follows since the intersection of the support of the two sets is disjoint. The proof of the second part is similar.

In the proofs of the mixing properties, we will need some observations about composite functions. Let $f_{\infty}=f\left(x_{r_{1}}, x_{r_{1}+1}, \ldots, x_{s_{1}}\right)$ and $g_{\infty}=g\left(x_{r_{2}}, x_{r_{2}+1}, \ldots, x_{s_{2}}\right)$. Then

$$
\begin{gathered}
f_{\infty} \circ g_{\infty}(x)_{i}=f_{\infty}\left(\ldots, g\left(x_{r_{2}-1}, \ldots, x_{s_{2}-1}\right), g\left(x_{r_{2}}, \ldots, x_{s_{2}}\right), g\left(x_{r_{2}+1}, \ldots, x_{s_{2}+1}\right), \ldots\right)_{i} \\
(6.3) \quad f\left(g\left(x_{r_{2}+r_{1}+i}, \ldots, x_{s_{2}+r_{1}+i}\right), g\left(x_{r_{2}+r_{1}+i+1}, \ldots, x_{s_{2}+r_{1}+i+1}\right), \ldots\right. \\
\left.\ldots, g\left(x_{r_{2}+s_{1}+i-1}, \ldots, x_{s_{2}+s_{1}+i-1}\right), g\left(x_{r_{2}+s_{1}+i}, \ldots, x_{s_{2}+s_{1}+i}\right)\right) .
\end{gathered}
$$

It follows that there exists some $h \in C\left(\mathbb{L}^{s_{1}+s_{2}-r_{1}-r_{2}}, \mathbb{L}\right)$ such that $h_{\infty}=f_{\infty} \circ$ $g_{\infty}=h\left(x_{r_{1}+r_{2}}, \ldots, x_{s_{1}+s_{2}}\right)$. In particular, if $f_{\infty}=f\left(x_{r}, \ldots, x_{s}\right)$, we have $f_{\infty}^{n}=$ $g\left(x_{n r}, \ldots, x_{n s}\right)$. We also observe that if $f$ is permutative in $x_{r_{1}}$, respectively $x_{s_{1}}$, and $g$ is permutative in $x_{r_{2}}$, respectively $x_{s_{2}}$, then $h$ is permutative in $x_{r_{1}+r_{2}}$, respectively $x_{s_{1}+s_{2}}$. 
Recall that a measure-theoretic dynamical system $(\Omega, \mu, \psi)$ is called strongly mixing if we for every measurable $A, B \subseteq \Omega$ have

$$
\lim _{n \rightarrow \infty} \mu\left(\psi^{-n}(A) \cap B\right)=\mu(A) \mu(B) .
$$

Theorem 6.2. Let $f_{\infty}=f\left(x_{r}, \ldots, x_{s}\right)$. If $s<0$ or $r>0$, then $\left(\mathbb{L}^{\infty}, \mu, f_{\infty}\right)$ is strongly mixing.

Proof. By Theorem 1.17 in [Wal] it is sufficient to prove equation (6.4) for all cylinders $A, B \subset \mathbb{L}^{\infty}$, since the elementary family of cylinder sets generates the Borel $\sigma$-algebra on $\mathbb{L}^{\infty}$. Let $A=\operatorname{Cyl}(x, p), B=\operatorname{Cyl}(y, q)$. Now $f_{\infty}^{n}=g\left(x_{n r}, \ldots, x_{n s}\right)$, thus

$$
\operatorname{Supp}\left(f_{\infty}^{-n}(A)\right)=\operatorname{Supp}\left(\left(f_{\infty}^{n}\right)^{-1}(\operatorname{Cyl}(x, p))\right) \subseteq[p+n r, p+n s+|x|-1] .
$$

If $r>0$, choose $n$ so large that $p+n r \geq q+|y|$, and if $s<0$, choose $n$ so large that $p+n s+|x|-1<q$. In both cases $\operatorname{Supp}\left(f_{\infty}^{-n}(A)\right) \cap \operatorname{Supp}(B)=\emptyset$, which implies that

$$
\mu\left(f_{\infty}^{-n}(A) \cap B\right)=\mu\left(f_{\infty}^{-n}(A)\right) \cdot \mu(B)=\mu(A) \mu(B) .
$$

Theorem 6.3. Let $f_{\infty}=f\left(x_{r}, \ldots, x_{s}\right)$. If $f$ is permutative in $x_{r}$ and $r<0$ or $f$ is permutative in $x_{s}$ and $s>0$, then $\left(\mathbb{L}^{\infty}, \mu, f_{\infty}\right)$ is strongly mixing.

Proof. Suppose $s>0$ and $f$ permutative in $x_{s}$. Let $A=\operatorname{Cyl}(x, p), B=\operatorname{Cyl}(y, q)$. Now $f_{\infty}^{n}=g\left(x_{n r}, \ldots, x_{n s}\right)$. Choose $n$ such that $p+n s-1 \geq q+|y|$. Then $\operatorname{Supp}(B) \subseteq(-\infty, p+n s-1]$. It follows from Lemma 6.1 that

$$
\mu\left(f_{\infty}^{-n}(A) \cap B\right)=\mu\left(f_{\infty}^{-n}(A)\right) \cdot \mu(B)=\mu(A) \mu(B) .
$$

The second part is analogous.

Corollary 6.4. Let $f_{\infty}=f\left(x_{r}, \ldots, x_{s}\right)$. If $f$ is permutative in both $x_{r}$ and $x_{s}$, then $\left(\mathbb{L}^{\infty}, \mu, f_{\infty}\right)$ is strongly mixing if and only if $r \neq 0$ or $s \neq 0$.

Proof. If $s<0$ or $r>0$ Theorem 6.2 applies. If $s>0$ or $r<0$ Theorem 6.3 applies. The only option left is $r=s=0$, in which case $f_{\infty}$ is clearly not strongly mixing.

Remark 6.5. Theorems 6.2 and 6.3 and Corollary 6.4 can also be found in [Sh-Ro, Theorems 3.2 and 3.4, and Corollary 3.5], but only for $S=2$.

A dynamical system $(\Omega, \mu, \psi)$ is called $m$-mixing if we for every measurable $A_{0}, A_{1}, \ldots, A_{m}$ have that

$$
\begin{gathered}
\lim _{k_{1}, k_{2}, \ldots, k_{m} \rightarrow \infty} \mu\left(A_{0} \cap \psi^{-k_{1}}\left(A_{1}\right) \cdots \cap \psi^{-\left(k_{1}+k_{2}+\cdots+k_{m}\right)}\left(A_{m}\right)\right) \\
=\mu\left(A_{1}\right) \mu\left(A_{2}\right) \cdots \mu\left(A_{m}\right) .
\end{gathered}
$$

It is evident that 1-mixing is strongly mixing. The converse is not a general fact, but we have the following direct generalization of Theorem 6.3:

Theorem 6.6. Let $f_{\infty}=f\left(x_{r}, \ldots, x_{s}\right)$. If $f$ is permutative in $x_{r}$ and $r<0$ or $f$ is permutative in $x_{s}$ and $s>0$, then $\left(\mathbb{L}^{\infty}, \mu, f_{\infty}\right)$ is $m$-mixing for all $m \in \mathbb{N}$. 
Proof. It is sufficient to prove the assertion for all cylinders $A_{0}, A_{1}, \ldots, A_{m}$. Choose $t$ such that $\operatorname{Supp}\left(A_{j}\right) \subseteq[-p, p]$ for all $j \in\{0,1, \ldots, m\}$. Suppose $f$ is permutative in $x_{s}$ and $s>0$. If $A_{j}=\operatorname{Cyl}\left(x^{(j)}, p_{j}\right)$, then $-p \leq p_{j} \leq p_{j}+\left|x^{(j)}\right|-1 \leq p$. Since for each $j \in \mathbb{N}$ there exists a function $g_{j}$ such that

$$
f_{\infty}^{k_{1}+k_{2}+\cdots+k_{j}}=g_{j}\left(x_{\left(k_{1}+k_{2}+\cdots+k_{j}\right) r}, \ldots, x_{\left(k_{1}+k_{2}+\cdots+k_{j}\right) s}\right),
$$

we obtain

$$
\begin{aligned}
\operatorname{Supp}\left(f_{\infty}^{-\left(k_{1}+\cdots+k_{j}\right)}\left(A_{j}\right)\right)=\operatorname{Supp}\left(\left(f_{\infty}^{k_{1}+\cdots+k_{j}}\right)^{-1}\left(A_{j}\right)\right) \\
\\
\subseteq\left[p_{j}+\left(k_{1}+\cdots+k_{j}\right) r, p_{j}+\left(k_{1}+\cdots+k_{j}\right) s+\left|x^{(j)}\right|-1\right] \\
\subseteq\left[-p+\left(k_{1}+\cdots+k_{j}\right) r, p+\left(k_{1}+\cdots+k_{j}\right) s-1\right] .
\end{aligned}
$$

Choose $k_{j}$ so large that $k_{j} s-1 \geq 2 p$ for all $j \in\{1,2, \ldots, m\}$. This implies that

$$
\begin{aligned}
\operatorname{Supp}\left(A_{0} \cap\right. & \left.f_{\infty}^{-k_{1}}\left(A_{1}\right) \cap f_{\infty}^{-\left(k_{1}+k_{2}\right)}\left(A_{2}\right) \cap \cdots \cap f_{\infty}^{-\left(k_{1}+k_{2}+\cdots+k_{j-1}\right)}\left(A_{j-1}\right)\right) \\
& \subseteq\left(-\infty, p+\left(k_{1}+\cdots+k_{j-1}\right) s\right] \\
& \subseteq\left(-\infty, p+\left(k_{1}+\cdots+k_{j-1}\right) s+k_{j} s-2 p-1\right] \\
& \subseteq\left(-\infty,-p+\left(k_{1}+\cdots+k_{j}\right) s-1\right] .
\end{aligned}
$$

The above equation together with Lemma 6.1 implies that

$$
\begin{aligned}
& \mu\left(A_{0} \cap f_{\infty}^{-k_{1}}\left(A_{1}\right) \cap f_{\infty}^{-\left(k_{1}+k_{2}\right)}\left(A_{2}\right) \cap \cdots\right. \\
& \left.\cdots \cap f_{\infty}^{-\left(k_{1}+k_{2}+\cdots+k_{j-1}\right)}\left(A_{j-1}\right) \cap f_{\infty}^{-\left(k_{1}+k_{2}+\cdots+k_{j}\right)}\left(A_{j}\right)\right) \\
& =\mu\left(A_{0} \cap f_{\infty}^{-k_{1}}\left(A_{1}\right) \cap \cdots \cap f_{\infty}^{-\left(k_{1}+k_{2}+\cdots+k_{j-1}\right)}\left(A_{j-1}\right)\right) \\
& \cdot \mu\left(f_{\infty}^{-\left(k_{1}+k_{2}+\cdots+k_{j}\right)}\left(A_{j}\right)\right) .
\end{aligned}
$$

Since $f_{\infty}$ is measure-preserving, the statement of the theorem follows by induction on $m$. If $f$ is permutative in $x_{r}$ and $r<0$ the proof is analogous.

The above mixing results are not complete. Shirvani and Rogers suggest in [Sh-Ro] that every onto or equivalently measure-preserving $f_{\infty}=f\left(x_{r}, \ldots, x_{s}\right)$ where $s>r$ is strongly mixing. If this conjecture is true, I believe it is difficult to prove in general.

\section{Measure-TheORETIC AND TOPOLOGICAL ISOMORPHISM}

Let $\mathbb{Z}_{n}$ be the ring of $n$ integers with discrete topology and normalized Haarmeasure. Define $\mathbb{Z}_{n}^{\infty}=\prod_{m=1}^{\infty} \mathbb{Z}_{n}$ and equip $\mathbb{Z}_{n}^{\infty}$ with product topology and product measure $\nu$. Let $\sigma$ be the left shift on $\mathbb{Z}_{n}^{\infty}$. It is easy to see that $\sigma$ is measurepreserving, since we only need to consider cylinder sets. This implies that the triple $\left(\mathbb{Z}_{n}^{\infty}, \nu, \sigma\right)$ is a dynamical system.

We want to show that a dynamical system $\left(\mathbb{L}^{\infty}, \mu, f_{\infty}\right)$, where $f_{\infty}=f\left(x_{r}, \ldots, x_{s}\right)$ and $r<0<s$ and $f$ is permutative in both $x_{r}$ and $x_{s}$, is measure-theoretic isomorphic to $\left(\mathbb{Z}_{S^{s-r}}^{\infty}, \sigma, \nu\right)$. 
Theorem 7.1. Let $f_{\infty}=f\left(x_{r}, \ldots, x_{s}\right)$, and suppose that $f$ is permutative in both $x_{r}$ and $x_{s}$ and further that $r<0<s$. Then there exists a collection $\left\{\varphi_{\kappa}: \kappa \in\right.$ $\left.\mathbb{L}^{s-r}\right\}: \mathbb{L}^{\infty} \rightarrow \mathbb{L}^{\infty}$ of cross-sections such that $\mathbb{L}^{\infty}=\bigcup_{\kappa \in \mathbb{L}^{s-r}} \varphi_{\kappa}\left(\mathbb{L}^{\infty}\right), \varphi_{\kappa}\left(\mathbb{L}^{\infty}\right) \cap$ $\varphi_{\kappa^{\prime}}\left(\mathbb{L}^{\infty}\right)=\emptyset$ for $\kappa \neq \kappa^{\prime}$, and such that every clopen set in $\mathbb{L}^{\infty}$ is a finite union of sets $\varphi_{\kappa_{1}} \varphi_{\kappa_{2}} \cdots \varphi_{\kappa_{n}}\left(\mathbb{L}^{\infty}\right)$. Moreover, $\mu\left(\varphi_{\kappa_{1}} \varphi_{\kappa_{2}} \cdots \varphi_{\kappa_{n}}\left(\mathbb{L}^{\infty}\right)\right)=S^{-n(s-r)}$.

Proof. We saw in the proof of Theorem 3.2 that every $\kappa \in \mathbb{L}^{s-r}$ gives rise to a unique $y^{(\kappa)} \in f_{\infty}^{-1}(x)$ such that $y_{1}^{(\kappa)} y_{2}^{(\kappa)} \ldots y_{s-r}^{(\kappa)}=\kappa$. This implies that we can define $\varphi_{\kappa}(x)=f_{\infty}^{-1}(x) \cap \operatorname{Cyl}(\kappa, 0)$. Then $\varphi_{\kappa}$ is well-defined, one to one, and continuous.

The union and intersection properties are trivial. To prove that every clopen set has the required form, we claim that for each finite sequence $\kappa_{1}, \kappa_{2}, \ldots, \kappa_{n}$ there exist $z^{(n)} \in \mathbb{L}^{n(s-r)}$ such that

$$
\varphi_{\kappa_{1}} \varphi_{\kappa_{2}} \cdots \varphi_{\kappa_{n}}\left(\mathbb{L}^{\infty}\right)=\operatorname{Cyl}\left(z^{(n)},(n-1) r\right) .
$$

We prove the statement by induction on $n$. The statement is obviously right for $n=1$, since $\varphi_{\kappa}\left(\mathbb{L}^{\infty}\right)=\operatorname{Cyl}(\kappa, 0)$. For the induction-step we write

$$
\begin{aligned}
\varphi_{\kappa_{1}} \varphi_{\kappa_{2}} \cdots \varphi_{\kappa_{n}} \varphi_{\kappa_{n+1}}\left(\mathbb{L}^{\infty}\right) & =\varphi_{\kappa_{1}}\left(\operatorname{Cyl}\left(z^{(n)},(k-1) r\right)\right) \\
& =f_{\infty}^{-1}\left(\operatorname{Cyl}\left(z^{(n)},(k-1) r\right)\right) \cap \operatorname{Cyl}\left(\kappa_{1}, 0\right) .
\end{aligned}
$$

By remark 3.6 there exists a unique $z^{(n+1)} \in \mathbb{L}^{\left|z^{(n)}\right|+s-r}=\mathbb{L}^{(n+1)(s-r)}$ such that

$$
f_{\infty}^{-1}\left(\operatorname{Cyl}\left(z^{(n)},(n-1) r\right)\right) \cap \operatorname{Cyl}\left(\kappa_{1}, 0\right)=\operatorname{Cyl}\left(z^{(n+1)},(n-1) r+r\right) .
$$

Since every clopen set $A$ has finite support, we can find $p \in \mathbb{N}$ such that $\operatorname{Supp}(A) \subseteq$ $[-p, p]$. Choose $n$ so large that $(n-1) r \leq-p$ and $(n-1) s \geq p$. Then $A$ is a finite union of cylinders $\operatorname{Cyl}\left(z^{(n)},(n-1) r\right)$, thus every clopen set is a finite union of sets of the form $\varphi_{\kappa_{1}} \varphi_{\kappa_{2}} \cdots \varphi_{\kappa_{n}}\left(\mathbb{L}^{\infty}\right)$.

Since $\varphi_{\kappa_{1}} \varphi_{\kappa_{2}} \cdots \varphi_{\kappa_{n}}\left(\mathbb{L}^{\infty}\right)$ is a cylinder of length $n(s-r)$, it follows that this set has measure $S^{-n(s-r)}$.

Theorem 7.2. Let $\Omega$ be a compact, totally disconnected Hausdorff-space, and let $\psi: \Omega \rightarrow \Omega$ be continuous, onto and $n-1$. Suppose there exists, for each $j \in \mathbb{Z}_{n}$, a map $\varphi_{j}: \Omega \rightarrow \Omega$ such that $\psi \circ \varphi_{j}=\mathrm{id}, \Omega=\bigcup_{j \in \mathbb{Z}_{n}} \varphi_{j}(\Omega), \varphi_{i}(\Omega) \cap \varphi_{j}(\Omega)=\emptyset$ for $i \neq j$, and such that every clopen set in $\Omega$ is a finite union of sets $\varphi_{j_{1}} \varphi_{j_{2}} \cdots \varphi_{j_{k}}(\Omega)$. Then $(\Omega, \psi)$ is topologically isomorphic to $\left(\mathbb{Z}_{n}^{\infty}, \sigma\right)$. If in addition $\mu$ is the measure on $\Omega$ such that $\mu\left(\varphi_{j_{1}} \varphi_{j_{2}} \cdots \varphi_{j_{k}}(\Omega)\right)=n^{-k}$ for all $k \in \mathbb{N}$, then $(\Omega, \mu, \psi)$ is measuretheoretic isomorphic to $\left(\mathbb{Z}_{n}^{\infty}, \nu, \sigma\right)$.

Proof. Define the map $\Gamma: \Omega \rightarrow \mathbb{Z}_{n}^{\infty}$ by

$$
\Gamma(x)=j_{1}, j_{2}, j_{3}, \ldots \quad \text { if } \quad x \in \varphi_{j_{1}} \varphi_{j_{2}} \cdots \varphi_{j_{m}}(\Omega) \quad \forall m \in \mathbb{N} .
$$

For each $m \in \mathbb{N}$ can find unique $j_{1}, j_{2}, \ldots, j_{m} \in \mathbb{Z}_{n}$ such that $x \in \varphi_{j_{1}} \varphi_{j_{2}} \cdots \varphi_{j_{m}}(\Omega)$, since $\Omega$ equals the disjoint union $\bigcup_{j_{1}, \ldots, j_{m}=1}^{n}(\Omega)$ for all $m \in \mathbb{N}$. Since $\varphi_{j_{m+1}}(\Omega) \subset \Omega$, we have the inclusion $\varphi_{j_{1}} \varphi_{j_{2}} \cdots \varphi_{j_{m}} \varphi_{j_{m+1}}(\Omega) \subseteq \varphi_{j_{1}} \varphi_{j_{2}} \cdots \varphi_{j_{m}}(\Omega)$. Thus for each $x$ there is one and only one sequence $j_{1}, j_{2}, \ldots$ such that $x \in \varphi_{j_{1}} \varphi_{j_{2}} \cdots \varphi_{j_{m}}(\Omega)$ for all $m \in \mathbb{N}$. It follows that $\Gamma$ is well-defined. $\Gamma$ is surjective since the intersection of every decreasing sequence of closed sets in a compact space is nonempty.

If $x, y \in \Omega$ and $x \neq y, x$ and $y$ can be separated by two clopen sets $U_{1}$ and $U_{2}$, since $\Omega$ is a totally disconnected Hausdorff-space. Thus $x$ and $y$ can be separated 
by sets $\varphi_{j_{1}} \varphi_{j_{2}} \cdots \varphi_{j_{m}}(\Omega)$ and $\varphi_{k_{1}} \varphi_{k_{2}} \cdots \varphi_{k_{m^{\prime}}}(\Omega)$, since $U_{1}$ and $U_{2}$ are finite unions of such. It follows that $\Gamma$ is injective.

The topology on $\mathbb{Z}_{n}^{\infty}$ is generated by sets

$$
U_{j, k}=\prod_{n=1}^{k} \mathbb{Z}_{n} \times\{j\} \times \prod_{n=k+2}^{\infty} \mathbb{Z}_{n} .
$$

It is easy to see that $\Gamma^{-1}\left(U_{j, k}\right)=\psi^{-k} \circ \varphi_{j}(\Omega)$, which is open since $\psi$ is continuous. Thus $\Gamma$ is continuous, and hence a homeomorphism, since $\Omega$ is compact and $\mathbb{Z}_{n}^{\infty}$ is Hausdorff.

Suppose $\Gamma(x)=j_{1}, j_{2}, \ldots \in \mathbb{Z}_{n}^{\infty}$. Then

$$
\begin{aligned}
\Gamma \circ f_{\infty}(x) & =\Gamma\left(f_{\infty}\left(\varphi_{j_{1}} \varphi_{j_{2}} \cdots(\Omega)\right)\right)=\Gamma\left(\varphi_{j_{2}} \varphi_{j_{3}} \cdots(\Omega)\right) \\
& =\sigma\left(j_{1}, j_{2}, \ldots\right)=\sigma \circ \Gamma(x) .
\end{aligned}
$$

It remains to show that $\Gamma$ is invertible measure-preserving. We have that $U_{k}=$ $\prod_{m=1}^{k}\left\{j_{m}\right\} \times \prod_{m=k+1}^{\infty} \mathbb{Z}_{n}$ is an elementary family that generates the Borel- $\sigma$-algebra on $\mathbb{Z}_{n}^{\infty}$. Since

$$
\mu\left(\Gamma^{-1}\left(U_{k}\right)\right)=\mu\left(\varphi_{j_{1}} \varphi_{j_{2}} \cdots \varphi_{j_{k}}(\Omega)\right)=n^{-k}=\nu\left(U_{k}\right),
$$

$\Gamma$ is measure-preserving. Conversely, since $\Omega$ is totally disconnected and every clopen set is a finite union of sets $\varphi_{j_{1}} \varphi_{j_{2}} \cdots \varphi_{j_{k}}(\Omega)$, these sets form an elementary family that generates the $\sigma$-algebra on $\Omega$. Since

$$
\Gamma\left(\varphi_{j_{1}} \varphi_{j_{2}} \cdots \varphi_{j_{k}}(\Omega)\right)=\prod_{n=1}^{k}\left\{j_{k}\right\} \times \prod_{n=k+1}^{\infty} \mathbb{Z}_{n}
$$

and the sets on each side of the equation has the same measure $n^{-k}, \Gamma^{-1}$ is measurepreserving.

Corollary 7.3. Assume that $r<0<s, f_{\infty}=f\left(x_{r}, \ldots, x_{s}\right)$ where $f$ is permutative in both $x_{r}$ and $x_{s}$. It follows that the system $\left(\mathbb{L}^{\infty}, f_{\infty}\right)$ is topologically isomorphic to $\left(\mathbb{Z}_{S^{s-r}}^{\infty}, \sigma\right)$ and that the system $\left(\mathbb{L}^{\infty}, \mu, f_{\infty}\right)$ is measure-theoretically isomorphic to $\left(\mathbb{Z}_{S^{s-r}}^{\infty}, \nu, \sigma\right)$.

Proof. All requirements of Theorem 7.2 are covered by Theorem 7.1.

It would be nice if one could characterize dynamical systems arising from functions that are not permutative in both the first and last variable. But in this case the structure of the inverses can be more complicated, even if $f$ is very simple. I include (without proof) a theorem stating this.

Theorem 7.4. Let $f_{\infty}=f\left(x_{1}, x_{2}, x_{3}\right)$ be defined by $f(a, b, c)=a+b+c+b c$ $\bmod 2$. Let $\mathbb{L}_{n}^{\infty}=\left\{x \in \mathbb{L}^{\infty}:\right.$ Card $\left.f_{\infty}^{-1}(x)=n\right\}$. If there exists $p \in \mathbb{N}$ such that that $x_{m}=1$ for all $m>p$, then $x \in \mathbb{L}_{3}^{\infty}$. If for all $p \in \mathbb{N}$ there exist $m>p$ and $k \geq 0$ such that $01^{3 k+1} 0=x_{m} x_{m+1} \ldots x_{m+3 k+2}$, then $x \in \mathbb{L}_{1}^{\infty}$. Otherwise $x \in \mathbb{L}_{2}^{\infty}$.

\section{8. $C^{*}$-ALGEBRAS ASSOCIATED TO DYNAMICAL SYSTEMS}

Let $(\Omega, \mu, \sigma)$ be a dynamical system where $\Omega$ is a compact Hausdorff-space, and consider the Hilbert-space $\mathcal{H}=L^{2}(\Omega, \mu)$. Every $f \in C(\Omega)$ induces an operator $T_{f}$ given by $T_{f} \xi(x)=f(x) \xi(x)$. Then $\left\|T_{f}\right\|=\operatorname{ess} \sup _{x \in \Omega}|f(x)|=\|f\|_{\infty}$. The compactness of $\Omega$ implies that $\left\|T_{f}\right\|=\|f\|_{\infty}$ is finite. 
The transformation $\sigma$ induces an operator $V \in B(\mathcal{H})$ given by $V \xi(x)=\xi(\sigma x)$. Using that $\sigma$ is measure-preserving, we obtain that $V$ is an isometry.

Define $C^{*}(C(\Omega), \sigma)$ as the $C^{*}$-algebra generated by the operators $\left\{T_{f}: f \in\right.$ $C(\Omega)\}$ and the operator $V$ induced by $\sigma$. If the dynamical systems $(\Omega, \mu, \sigma)$ and $(\hat{\Omega}, \hat{\mu}, \hat{\sigma})$ are measure-theoretic and topologically isomorphic, $C^{*}(C(\Omega), \sigma)$ is isomorphic to $C^{*}(C(\hat{\Omega}), \hat{\sigma})$.

Theorem 8.1. Consider the dynamical system $\left(\mathbb{Z}_{n}^{\infty}, \nu, \sigma\right)$. Then $C^{*}\left(C\left(\mathbb{Z}_{n}^{\infty}\right), \sigma\right)$ is isomorphic to $\mathcal{O}_{n}$, the Cuntz-algebra of order $n$.

Proof. We denote $\mathbb{Z}_{n}^{\infty}$ by $\Omega$. Let $\sigma_{j}$ where $j \in \mathbb{Z}_{n}$ be the cross-sections of $\sigma$ given by $\sigma_{j}\left(x_{1} x_{2} x_{3} \ldots\right)=\left(j x_{1} x_{2} x_{3} \ldots\right)$. Let $\Omega_{j}=\sigma_{j}(\Omega)$ and $\Omega_{j_{1}, j_{2}, \ldots, j_{k}}=\sigma_{j_{1}} \sigma_{j_{2}} \cdots \sigma_{j_{k}}(\Omega)$, and let $P_{j_{1}, j_{2}, \ldots, j_{k}}$ be the projection on $\Omega_{j_{1}, j_{2}, \ldots, j_{k}}$.

Define the Cuntz-generators $S_{j}$ where $j \in \mathbb{Z}_{n}$ by

$$
S_{j}^{*} \xi(x)=n^{-\frac{1}{2}}\left(\xi \circ \sigma_{j}\right)(x), \quad \xi \in \mathcal{H}, \quad x \in \Omega .
$$

Since $\nu$ is a product-measure on $\Omega$, we have $\nu\left(\sigma_{j}(A)\right)=n^{-1} \nu(A)$ for all measurable $A \subseteq \Omega$. By a straightforward calculation we obtain that $S_{j}=n^{\frac{1}{2}} P_{j} V$, thus $\left.C^{*}(C(\Omega), \sigma)\right)$ contains $C^{*}\left(\left\{S_{j}\right\}_{j \in \mathbb{Z}_{n}}\right)$. Moreover, one may verify that $P_{i} P_{j}=$ $\delta_{i j} S_{i} S_{j}^{*}$, thus the Cuntz-relations hold, and we may identify $C^{*}\left(\left\{S_{j}\right\}_{j \in \mathbb{Z}_{n}}\right)$ with $\mathcal{O}_{n}$.

Since $\sum_{j \in \mathbb{Z}_{n}} S_{j}=n^{\frac{1}{2}} V$, it follows that $V \in \mathcal{O}_{n}$. Moreover

$$
P_{j_{1}, j_{2}, \ldots, j_{k}}=S_{j_{1}} S_{j_{2}} \cdots S_{j_{k}} S_{j_{k}}^{*} \cdots S_{j_{2}}^{*} S_{j_{1}}^{*} .
$$

By the Stone-Weierstrass theorem, $\mathcal{O}_{n}$ contains $T_{f}$ for all $f \in C(\Omega)$, since each projection $P_{j_{1}, j_{2}, \ldots, j_{k}}$ is contained in $\mathcal{O}_{n}$. Thus $C^{*}(C(\Omega), \sigma) \subseteq \mathcal{O}_{n}$. See also $[\mathrm{Br}-\mathrm{J} \varnothing-\mathrm{Pr}]$.

Corollary 8.2. Assume that $r<0<s, f_{\infty}=f\left(x_{r}, \ldots, x_{s}\right)$ where $f$ is permutative in both $x_{r}$ and $x_{s}$. It follows that $C^{*}\left(C\left(\mathbb{L}^{\infty}\right), f_{\infty}\right)$ is isomorphic to $\mathcal{O}_{n}$.

Proof. This follows from Theorem 8.1 and Corollary 7.3.

Remark 8.3. Kengo Matsumoto has obtained less general results in [Mat]. He has proved the above corollary for $f_{\infty}=f\left(x_{-1}, x_{0}, x_{1}\right)=x_{-1}+x_{1}$ mod 2 in Theorem 3.14 and for $f_{\infty}=f\left(x_{-1}, x_{0}, x_{1}\right)=x_{-1}+x_{0}+x_{1} \bmod 2$ in Proposition 3.16.

\section{REFERENCES}

[Br-Jø-Pr] Ola Bratteli, Palle E. T. Jørgensen and Geoffrey L. Price. Endomorphisms of B(H). Proc. Symp. Pure Math. Am. math. soc. 1995. (ed. I. E. Segal.) To appear.

[Cuntz] Joachim Cuntz, Simple $C^{*}$-algebras generated by isometries. Commun. Math. Phys. 57, 173-185 (1977). MR 57:7189

[Hed] G. A. Hedlund. Endomorphisms and automorphisms of the shift dynamical system. Math. Syst. theory 3, 320-375 (1970). MR 41:4510

[Mat] Kengo Matsumoto. $C^{*}$-algebras associated width dynamical systems. Math. Scand. 75, 195-216 (1994). MR 96a:46120

[Sh-Ro] M. Shirvani and T. D. Rogers. On ergodic one-Dimensional Cellular Automata, Commun. Math. Phys. 136, 599-605 (1991). MR 92j:58057

[Wal] Peter Walters. An introduction to ergodic theory. Springer (1982). MR 84e:28017

Department of Mathematics, University of Oslo, Box 1053, 0316 Oslo, Norway

E-mail address: runekl@math.uio.no 